\title{
Highly Tunable Electrothermally and Electrostatically Actuated Resonators
}

\author{
Amal Z. Hajjaj, Nouha Alcheikh, Abdallah Ramini, Md Abdullah Al Hafiz and Mohammad I. Younis ${ }^{1}$
}

\begin{abstract}
This paper demonstrates experimentally, theoretically, and numerically, for the first time, a widerange tunability of an in-plane clamped-clamped microbeam, bridge, resonator actuated electrothermally and electrostatically. Using both actuation methods, we demonstrate that a single resonator can be operated at a wide range of frequencies. The microbeam is actuated electrothermally, by passing a DC current through it, and electrostatically by applying a DC polarization voltage between the microbeam and the stationary electrode. We show that when increasing the electrothermal voltage, the compressive stress inside the microbeam increases, which leads eventually to its buckling. Before buckling, the fundamental frequency decreases until it drops to very low values, almost to zero. After buckling, the fundamental frequency increases, which is shown to be as high as twice the original resonance frequency. Adding a DC bias changes the qualitative nature of the tunability both before and after buckling, which adds another independent way of tuning. This reduces the dip before buckling, and can eliminate it if desired, and further increases the fundamental frequency after buckling. Analytical results based on the Galerkin discretization of the Euler Bernoulli beam theory are generated and compared to the experimental data and to simulation results of a multi-physics finite-element model. A good agreement is found among all the results.
\end{abstract}

Index Terms - Tunability, Resonator, Electrothermal Actuation, Electrostatic Actuation.

\section{INTRODUCTION}

$\mathrm{B}_{\mathrm{s}}^{\mathrm{i}}$ istable microelectromechanical (MEMS) structures have been drawing significant attention recently for their interesting advantages in application, such as energy harvesting [1], sensors [2], actuators [3], and MEMS/NEMS based memory

\footnotetext{
${ }^{1}$ Manuscript received December 24, 2015; revised February 18, 2016; accepted March 11, 2016. Subject Editor G. Piazza. The authors are with the Department of Mechanical Engineering, King Abdullah University of Science and Technology, Thuwal 239556900, Saudi Arabia (e-mail: amal.hajjaj@kaust.edu.sa; nouha.alcheikh@ kaust.edu.sa; abdallah.ramini@kaust.edu.sa; abdullah.hafiz@kaust.edu.sa; myounis@ binghamton.edu).

Color versions of one or more of the figures in this paper are available online at http://ieeexplore.ieee.org. Digital Object Identifier 10.1109/JMEMS.2016.2542338
}

elements [4]. Bistable microstructures are characterized by a double-well potential, and hence at least two stable states, and commonly a third one encircles the two local wells. The motion resulting from this third state is large compared with the other two in-well motion. A well- known example of this is the snap-through motion in buckled beams. This is highly desirable feature for many application including micro-mirrors [5], micro-switching [6], and micro-actuators [7]. Bistable microstructure can be realized in many configurations, such as beams sandwiched between two magnets, shallow arches, imperfect microbeams, and buckled beams. This paper is concerned with the third category. The buckled beam is mainly realized by an axial compressive load that can be induced by several methods, such as applying a direct axial load [8] or by using thermal actuation [9]. In this work, we study the generation of axial-compressive load using thermal expansion induced by Joule heating.

Joule heating is a common mechanism of actuation in MEMS thanks to its easy implementation. Joule heating is the conversion of the electrical current energy flowing through a structure into heat. The electrothermal voltage $V_{T h}$ is applied between the anchors of the microbeam, inducing the current $I_{T h}$ that passes through the microbeam and controls its internally induced axial stress caused by thermal expansion. Nonetheless, the elongation is prevented by the presence of the fixed anchors of the microbeam, which induces a compressive force. This compressive load can lead to bucking of the microbeam. This phenomenon can be analyzed from two aspects: electrothermal problem; describing the conversion of the electrical power into heat; and thermo-elastic problem; describing the conversion of the heat power into compressive stress.

Electrothermal actuation has been mainly used as a mechanism to achieve static buckling in microbeams. Chioa and Lin [10] studied theoretically and experimentally the critical current for a fixedfixed microbeam to buckle. Wang et al [11] developed an electrothermally actuated lateral contact microrelay for RF applications. They studied the required voltage for the microrelay utilizing the parallel six-beam. Chen et al [12] reported a theoretical and experimental investigation of the 
post-buckling behavior of electrothermally actuated beams. Mastropaolo and Cheung [13] investigated the behavior of $\mathrm{SiC}$ clamped-clamped bridge resonators electrothermally actuated with U-shaped aluminum electrodes on top as a function of electrode length, width, and spacing.

Among the few works that utilize electrothermal actuation for dynamic excitation, Sibgatullin et al [14] used the electrothermal excitation as a way to excite the parametric resonance of a clamped-clamed microbeam.

Electrothermal actuation is also widely used to excite and to tune compliant resonators. Tunability of Micro/Nano-electromechanical resonators is highly desirable feature for various applications, including communications [15], filtering [16], gyroscopes [17], energy harvesting [18], signal processing [19], and ultrasensitive detection [20]. At the Micro-scale, electrothermal actuation has been utilized to tune the resonance frequency of resonators, however for a very limited range of frequency. Remtema and Lin [21] showed experimentally and theoretically that the resonance frequency of a resistively heated microbeam could be reduced by $6.5 \%$. Goktas and Zaghloul [22] studied the tunability of CMOSMEMS fixed-fixed beam resonators using embedded heaters to create axial stress inside the resonator. They showed that the frequency could be decreased by $42.6 \%$. Sviličić et al [23] presented design, fabrication, and electrical testing of MEMS resonators actuated electrothermally and including a piezo-electric sensor to detect the resonance frequency of these resonators. They demonstrated that with the increase of the electrothermal actuation voltage a tuning range of $17 \mathrm{kHz}$ could be realized for a device resonating at $1.766 \mathrm{MHz}$. At the Nanoscale, the resonance frequency of electrothermally actuated nanomechanical resonators have been tuned for lower values [24], as demonstrated at the Microscale.

The use of electrostatic actuation to tune resonators is well known since the early work of Nathanson et al [25]. However, it does not offer wide range of frequency, and is mainly used to decrease the resonance frequency of resonators through the softening effect [26]; except for few studies that demonstrate that the resonance frequency could be increased marginally as tuning the electrostatic force for specific geometric conditions [27]. Nevertheless, at the Nano-scale recent experimental evidences demonstrated that the resonance frequency of Carbon Nano Tube CNT resonators increases considerably when increasing the DC gate voltage [28].

The combination of both electrothermal and electrostatic actuation has been rarely studied before, for example for MEMS cantilever switches [29]. No studies have been presented so far that exploit the full potential of these actuation mechanisms in achieving large range of tunability for resonators. This will be demonstrated in this work. The resonator under consideration is composed of an elastic clampedclamped straight microbeam hanging above a stationary electrode; actuated electrothermally by passing a DC current through it and electrostatically by applying a DC voltage between it and the stationary electrode. We aim to investigate theoretically, numerically, and experimentally the tunability, decreasing, and increasing the frequency of this bridge resonator as varying the electrothermal voltage and for several fixed DC polarization voltages.

The rest of the paper is organized as follows. The nonlinear Euler-Bernoulli beam equation combined with the heat conduction equation is solved for the pre-buckled and post-buckled behavior in Section II. Numerically, a multi-physics nonlinear finite element model, which takes into account the structural, electrothermal, and electrostatic domains, is described in Section III. The experimental setup is presented in Section IV. A discussion of the obtained measurements as compared to simulations is reported in Section V. Finally, the main conclusions are summarized in Section VI.

\section{PROBLEM FORMULATION}

The device under consideration, Fig. 1, consists of an in-plane clamped-clamped microbeam made of doped silicon and actuated electrothermally by a DC voltage $V_{T h}$ and electrostatically by a DC polarization voltage $V_{D C}$. It is subjected to a viscous damping of coefficient $\hat{c}$. This linearly elastic microbeam, with Young's modulus $E$ and material density $\rho$, is of length $l$, width $b$, and thickness $h$. It is assumed to have a rectangular cross section area $A=b h$ and a moment of inertia $I=b h^{3} / 12$. The microbeam is separated from a stationary electrode with a gap width $d$ and with a dielectric constant of the medium $\varepsilon$. The electrothermal voltage $V_{T h}$ is applied between the anchors of the microbeam inducing a current $I_{T h}$ passing through the microbeam that heats up it and controls its internally induced axial stress.

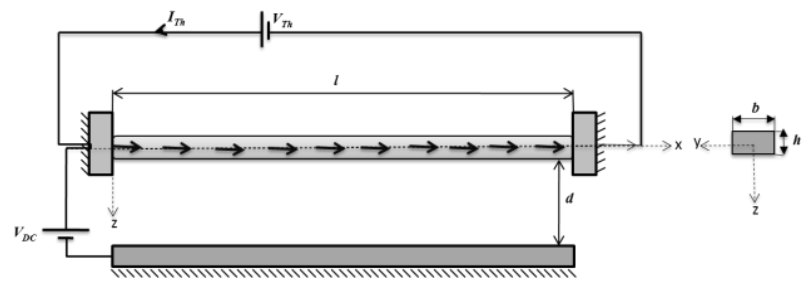

(a) 


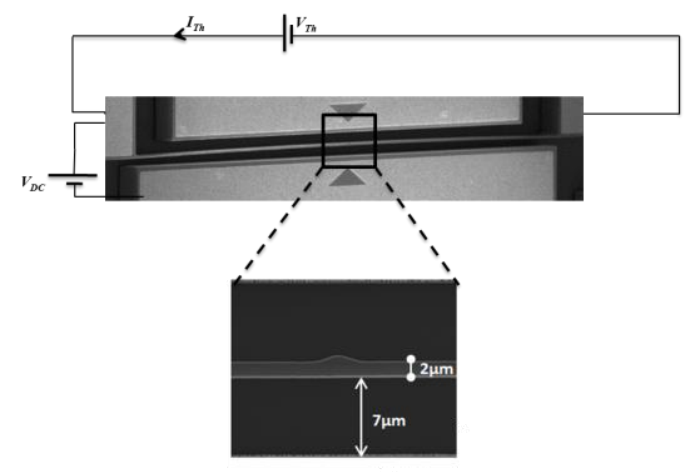

(b)

Fig. 1. (a) Schematic of an electrothermally and electrostatically actuated clamped-clamped microbeam. (b) Top view SEM picture of the buckled configuration of the actual device with a schematic of the actuation mechanism.

The assumed geometrical, mechanical, thermal and electrical parameters of the studied microbeam are shown in Table 1.

TABLE I.

GEOMETRICAL, MECHANICAL, THERMAL AND ELECTRICAL PROPERTIES OF THE MICROBEAM MADE OF DOPED SILICON.

\begin{tabular}{ccc}
\hline \hline Symbol & Quantity & Value \\
\hline$l$ & Length & $600 \mu \mathrm{m}$ \\
$b$ & Thickness & $2 \mu \mathrm{m}$ \\
$d$ & Width & $25 \mu \mathrm{m}$ \\
$E$ & Gap & $7 \mu \mathrm{m}$ \\
$\rho$ & Young's Modulus & $120 \mathrm{GPa}$ \\
$\alpha$ & Density & $2332 \mathrm{~kg} / \mathrm{m}^{3}$ \\
$k$ & Coefficient of Thermal & $2.610^{-6} \mathrm{~K}^{-1}$ \\
& Expansion & \\
$\sigma_{e}$ & Thermal Conductivity & $165 \mathrm{~W} /(\mathrm{m} \mathrm{K})$ \\
& Electrical Conductivity & $0.7810^{4} \mathrm{~S} / \mathrm{m}$ \\
\hline \hline
\end{tabular}

\section{A. Electrothermal problem}

Passing an electrical current through a conductor induces the so-called Joule heating, which is mainly caused by the interaction between the moving particles that form the electrical current and the atomic ions that make up the body of the conductor. These particles forming the electrical current give up some of their kinetic energy each time they collide with an ion. This kinetic energy induces the rise of the temperature inside the conductor, which transforms the electrical energy into thermal energy.

In the studied device of this work, applying a potential voltage across the anchors of the microbeam generates a heat flux of density $E=J^{2} / \sigma_{\mathrm{e}}$ per volume, where $J$ represents the current density defined by $I_{T h} / A$ and $\sigma_{\mathrm{e}}$ represents the electrical conductivity of the microbeam material. The current density is assumed to be uniformly distributed along the microbeam. In our study the convection and the thermal radiation of the microbeam are assumed negligible. Also, the deformation of the microbeam arising from the thermo-elastic coupling induced by the electric current is neglected. For simplification, thermal conductivity and electrical conductivity are assumed to be independent of temperature. Under all the above assumptions and referring to the heat equation; Fourier's law; the equation governing the average temperature across the section of the microbeam induced by the current $I_{T h}$ is given as below:

$$
-k \frac{d^{2} T}{d \hat{x}^{2}}=\frac{J^{2}}{\sigma_{e}}
$$

where $k$ is the thermal conductivity of the microbeam material. The current density can be written as a function of the DC electrothermal voltage $J=\frac{\sigma_{e} V_{T h}}{l}$. Therefore, the heat equation can be written as

$$
-k \frac{d^{2} T}{d \hat{x}^{2}}=\frac{\sigma_{e} V_{T h}^{2}}{l^{2}}
$$

Solving (2), assuming that the temperature at the ends of the microbeam is equal to the ambient temperature $T_{a}$, gives a close form solution of the distribution of the temperature along the microbeam, which has a parabolic shape and given by the below equation:

$$
T[\hat{x}]=\frac{\sigma_{e} V_{T h}^{2}}{2 k}\left(\frac{\hat{x}}{l}-\frac{\hat{x}^{2}}{l^{2}}\right)+T_{a}
$$

The variation of the temperature along the microbeam induces the thermal stress given by

$$
\hat{S}_{T h}=\alpha E A \int_{0}^{l}\left(T[\hat{x}]-T_{a}\right) d \hat{x}
$$

where $\alpha$ is the coefficient of thermal expansion, which is assumed here to be independent of temperature.

\section{B. Equation of Motion}

The governing equation of motion of the microbeam under consideration, Fig. 1, describing its 
transverse deflection $\hat{w}(\hat{x}, \hat{t})$ is written as follows [30]:

$$
\begin{aligned}
\rho b h \frac{\partial^{2} \hat{w}}{\partial \hat{t}^{2}}+\hat{c} & \frac{\partial \hat{w}}{\partial \hat{t}}+E I \frac{\partial^{4} \hat{w}}{\partial \hat{x}^{4}}= \\
& \frac{\partial^{2} \hat{w}}{\partial \hat{x}^{2}}\left[\hat{N}+\frac{E A}{2 l} \int_{0}^{l}\left(\frac{\partial \hat{w}}{\partial \hat{x}}\right)^{2} d \hat{x}\right] \\
& +\frac{1}{2} \varepsilon b \frac{\left(V_{D C}+V_{A C} \cos (\hat{\Omega} t)\right)^{2}}{(d-\hat{w})^{2}}
\end{aligned}
$$

The microbeam is subjected to the following boundary conditions:

$$
\begin{aligned}
& \hat{w}(0, \hat{t})=\hat{w}(l, \hat{t})=0 \\
& \left.\frac{d \hat{w}}{d \hat{x}}\right|_{(0, \hat{t})}=\left.\frac{d \hat{w}}{d \hat{x}}\right|_{(l, \hat{t})}=0
\end{aligned}
$$

where $\hat{x}$ is the position along the microbeam and $\hat{t}$ is time. The term $\hat{N}=\hat{N}_{0}-\hat{S}_{T h}$ represents the axial load due to the residual axial load, where $\hat{N}_{0}$ arising from the fabrication process and the compressive axial load and $\hat{S}_{T h}$ originated from the thermal stress induced by the electrical current $I_{T h}$ given by (4).

For convenience, we introduce the nondimensional variables as below:

$$
w=\frac{\hat{w}}{d} ; x=\frac{\hat{x}}{l} \text { and } t=\frac{\hat{t}}{T}
$$

where $T=\sqrt{\frac{\rho b h l^{4}}{E I}}$ is a time scale. Substituting (7) into (5) and (6), we obtain the nondimensional equation of motion of the beam

$$
\begin{aligned}
& \frac{\partial^{2} w}{\partial t^{2}}+c \frac{\partial w}{\partial t}+\frac{\partial^{4} w}{\partial x^{4}}= \\
& \frac{\partial^{2} w}{\partial x^{2}}\left[N_{0}-S_{T h}+\alpha_{1} \int_{0}^{1}\left(\frac{\partial w}{\partial x}\right)^{2} d x\right]+\alpha_{2} \frac{\left(V_{D C}+V_{A C} \cos (\Omega t)\right)^{2}}{(1-w)^{2}}
\end{aligned}
$$

Subjected to the nondimensional boundary conditions

$$
\begin{aligned}
& w(0, t)=w(1, t)=0 \\
& \left.\frac{d w}{d x}\right|_{(0, t)}=\left.\frac{d w}{d x}\right|_{(1, t)}=0
\end{aligned}
$$

The nondimensional (except for $\alpha_{2}$ ) parameters appearing in (8) are defined as below:

$$
\begin{aligned}
& \alpha_{1}=6\left(\frac{d}{h}\right)^{2} ; \alpha_{2}=\frac{6 \varepsilon l^{4}}{E h^{3} d^{3}} ; N_{0}=\frac{l^{2}}{E I} \hat{N}_{0} ; \\
& S_{T h}=\frac{l^{2}}{E I} \hat{S}_{T h} ; c=\frac{l^{4}}{E I T} \hat{c} \text { and } \Omega=T \hat{\Omega}
\end{aligned}
$$

Since the microbeam is subjected to a compressive load that increases as much as we increase the electrothermal voltage, it is expected that the microbeam encounters a pitchfork bifurcation near a critical load; below which the microbeam remained straight and above which the microbeam buckles. Therefore, next we split the problem into two parts: the pre-buckling behavior and the postbuckling behavior of the microbeam.

\section{Pre-Buckling Study}

In this part, the microbeam is modeled as a straight microbeam under a compressive axial load and electrostatic force. A reduced-order model is derived to compute the static deflection as well as the variation of the fundamental natural frequency while varying the electrothermal voltage $V_{T h}$ and for fixed values of the electrostatic voltage $V_{D C}[30,31]$.

To determine the static deflection $w_{s}(x)$ we set the time derivatives and the $\mathrm{AC}$ force equal to zero in (8):

$$
\begin{array}{r}
\frac{\partial^{4} w_{s}}{\partial x^{4}}=\left[N_{0}-S_{T h}+\alpha_{1} \int_{0}^{1}\left(\frac{\partial w_{s}}{\partial x}\right)^{2} d x\right] \frac{\partial^{2} w_{s}}{\partial x^{2}} \\
+\alpha_{2} \frac{V_{D C}{ }^{2}}{\left(1-w_{s}\right)^{2}}
\end{array}
$$

with the associated boundary conditions

$$
\begin{aligned}
& w_{s}(0)=w_{s}(1)=0 \\
& \left.\frac{d w_{s}}{d x}\right|_{x=0}=\left.\frac{d w_{s}}{d x}\right|_{x=1}=0
\end{aligned}
$$

To solve (11), we refer to the Galerkin procedure in which we use the undamped linear mode shapes of a straight unactuated microbeam as basis functions [30]. Therefore the deflection is expressed as

$$
w_{s}(x)=\sum_{i=0}^{n} a_{i} \phi_{i}(x)
$$

where $a_{i}(i=0,1,2 \ldots n)$ denotes the nondimensional modal static coefficients and $\phi_{i}(x)(i=0,1,2 \ldots n)$ denotes the undamped mode shapes of the straight unactuated beam governed by:

$$
\frac{d^{4} \phi_{i}}{d x^{4}}-\left(N_{0}-S_{T h}\right) \phi_{i}^{\prime \prime}-\omega_{i}^{2} \phi^{\prime \prime}=0
$$

With the associated boundary conditions: 


$$
\begin{aligned}
& \phi_{i}(0)=\phi_{i}(1)=0 \\
& \left.\frac{d \phi_{i}}{d x}\right|_{x=0}=\left.\frac{d \phi_{i}}{d x}\right|_{x=1}=0
\end{aligned}
$$

To determine the variation of the natural frequency of the microbeam under the electrostatic DC polarization voltage and various electrothermal voltages, we solve the eigenvalue problem obtained by perturbing the deflection around the static configuration. Toward this, we write

$$
w(x, t)=w_{s}(x)+v(x, t)
$$

We resort to the Galerkin discretization to represent the dynamic amplitude $v(x, t)$ as

$$
v(x, t)=\sum_{i=0}^{n} u_{i}(t) \phi_{i}(x)
$$

where $u_{i}(t)(i=0,1,2 . . n)$ denotes the nondimensional modal coordinates and $\phi_{i}(x)$ as defined in (14).

Then, we substitute (16) and (17) into (8), then subtract the equilibrium equitation, (11), from the outcome, multiplying the result by the mode shape $\phi_{j}$, applying the orthogonality condition of the mode shapes, and integrating over the beam domain (from 0 to 1 ), which yields the below equation [30]

$$
\begin{aligned}
\ddot{u}_{j}+u_{j} \omega_{j}{ }^{2}= & \\
& {\left[2 \alpha_{1} \int_{0}^{1}\left(\phi_{j} \frac{\partial^{2} w_{s}}{\partial x^{2}}\right) d x \int_{0}^{1}\left(\sum_{i=0}^{n} u_{i} \phi_{i} \frac{\partial w_{s}}{\partial x}\right) d x\right.} \\
+ & {\left[\alpha_{1} \int_{0}^{1}\left(\frac{\partial w_{s}}{\partial x}\right)^{2} d x\right]_{0}^{1}\left(\phi_{j}\left(\sum_{i=0}^{n} u_{i} \phi_{i}{ }^{\prime}\right)\right) d x } \\
+ & \int_{0}^{1}\left[\phi_{j} \frac{2 \alpha_{2} V_{D C}{ }^{2}}{\left(1-w_{s}\right)^{3}}\left(\sum_{i=0}^{n} u_{i} \phi_{i}\right)\right] d x
\end{aligned}
$$

Using three symmetric mode shapes, three linearized ordinary differential equations are obtained. For a given $V_{T h}$ and $V_{D C}$, we compute the Jacobin of the system and find the corresponding eigenvalues. Then, by taking the square root of these eigenvalues, we find the natural frequencies of the resonators under $V_{T h}$ and $V_{D C}$.

\section{Post-Buckling Study:}

The term "Post-buckling" here refers to the fact that the applied axial force exceeds the critical load of the case without electrostatic force. Essentially, the electrostatic force biases the beam, and hence the pitchfork bifurcation of the buckling instability becomes a perturbed pitchfork bifurcation. Here we use the buckled mode shapes and frequencies in the Galerkin discretization as well as the first buckled configuration, as developed by Nayfeh et al [32, 33] and presented in the appendix. We study the transverse vibration induced by the electrostatic force around the static buckled configuration. To do so, we split the static deflection induced by both thermal stress and electrostatic force, $w_{s, b}(x)$, into two components as follows

$$
w_{s, b}(x)=\psi(x)+\chi(x)
$$

where $\chi(x)$ is deflection induced by the electrostatic force and $\psi(x)$ is the buckled configuration given by (A3) in the appendix. Substituting (19) into (11) and subtracting the static equation for $\psi$, (A1) from the appendix, we obtain the governing equation of $\chi(x)$

$$
\begin{aligned}
& \chi^{i v}=\left[N_{0}-S_{T h}+\alpha_{1} \int_{0}^{1} \chi^{\prime 2} d x+\alpha_{1} \int_{0}^{1} \psi^{\prime 2} d x+2 \alpha_{1} \int_{0}^{1} \psi^{\prime} \chi^{\prime} d x\right] \chi^{\prime \prime} \\
& +\left[\alpha_{1} \int_{0}^{1} \chi^{\prime 2} d x+2 \alpha_{1} \int_{0}^{1} \psi^{\prime} \chi^{\prime} d x\right] \psi^{\prime \prime} \\
& +\alpha_{2} \frac{V_{D C}{ }^{2}}{(1-\psi-\chi)^{2}}
\end{aligned}
$$

With the associated boundary condition:

$$
\begin{aligned}
& \chi(0)=\chi(1)=0 \\
& \left.\frac{d \chi}{d x}\right|_{x=0}=\left.\frac{d \chi}{d x}\right|_{x=1}=0
\end{aligned}
$$

To solve (20), we use the Galerkin procedure with the undamped linear mode shapes of an unactuated (zero DC voltage) buckled beam, presented in the appendix (A8), as basis functions.

Next, we determine the variation of the natural frequency of the buckled microbeam under the DC polarization voltage and as varying the electrothermal voltage. Next we determine the variation of the natural frequency of the buckled microbeam at various electrostatic DC polarization voltage and electrothermal voltage. Thus,

$$
w(x, t)=w_{s, b}(x)+y(x, t)
$$

Then, the Galerkin discretization is used to represent the dynamic amplitude $y(x, t)$ as

$$
y(x, t)=\sum_{i=0}^{n} z_{i}(t) \varphi_{i}(x)
$$

where $z_{i}(t)(i=0,1,2 . . n)$ denotes the nondimensional modal coordinates and $\varphi_{i}(x)$ is as defined in (A5) in the appendix. 
Then, we substitute (22) and (23) into (8), multiplying the outcome by the mode shape $\varphi_{j}$, applying the orthogonality condition of the mode shapes, and integrating over the beam domain (from 0 to 1 ), which yields the below equation [28]:

$$
\begin{aligned}
\ddot{z}_{j}+z_{j} \omega_{j}{ }^{2}= & \\
& {\left[2 \alpha_{1} \int_{0}^{1}\left(\varphi_{j} \frac{\partial^{2} w_{s}}{\partial x^{2}}\right) d x\right] \int_{0}^{1}\left(\sum_{i=0}^{n} u_{i} \varphi_{i} \frac{\partial w_{s}}{\partial x}\right) d x } \\
+ & {\left[\alpha_{1} \int_{0}^{1}\left(\frac{\partial w_{s}}{\partial x}\right)^{2} d x\right]_{0}^{1}\left[\phi_{j}\left(\sum_{i=0}^{n} u_{i} \varphi_{i}^{\prime \prime}\right)\right) d x } \\
+ & \int_{0}^{1}\left[\varphi_{j} \frac{2 \alpha_{2} V_{D C}{ }^{2}}{\left(1-w_{s}\right)^{3}}\left(\sum_{i=0}^{n} u_{i} \varphi_{i}\right)\right] d x
\end{aligned}
$$

A three symmetric mode shapes are used to compute the Jacobin of the system and find the corresponding eigenvalues by taking the square root of these eigenvalues. Then, we find the natural frequencies of the resonators under $V_{T h}$ and $V_{D C}$.

\section{FINITE ELEMENT MODEL}

To further verify the analytical findings, and to assure that the various assumptions made in the analytical model are valid, we conduct a 3D multiphysics finite-element simulation of the clampedclamped microbeam. The analysis is done using the commercial finite element software COMSOL [34]. We have taken the same material parameters and geometric dimensions of Table I. To account for the various physical domains of the problem, the Solid Mechanics, Electric Currents, and Heat Transfer interfaces are considered. The anchors are assigned a fixed constraint boundary condition with ambient temperature at their bottom. The rest of the faces of the structure are set to a convective heat boundary condition, where the heat flux option is used for an external natural convection with air as an external fluid and a vertical wall height of $1 \mathrm{~m}$. For the Electric Currents module, an electrical potential and a ground were defined on the top of the anchors to allow passing an electrical current trough a conductor and simulate the Joule heating. Fig. 2 shows the thermal distribution and the displacement corresponding to $V_{T h}=3.5 \mathrm{~V}$ and with no electrostatic polarization voltage.

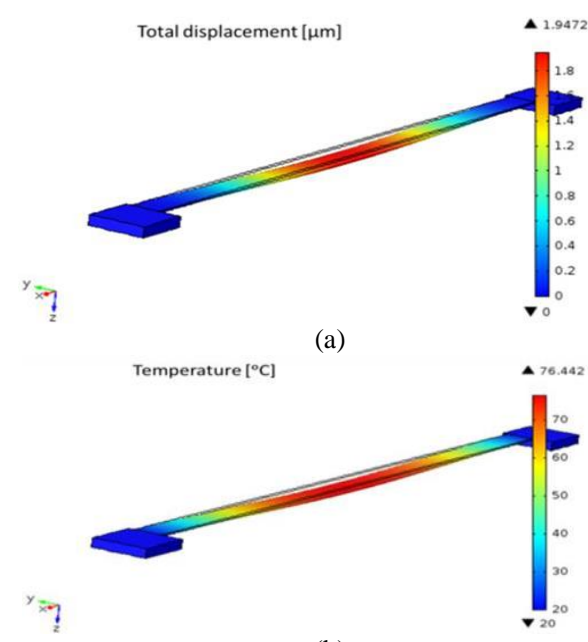

(b)

Fig. 2. (a) Total displacement. (b)Temperature distribution at $V_{T h}=3.5 \mathrm{~V}$.

To simulate a clamped-clamped microbeam under compressive load induced by the electrothermal voltage and under the electrostatic force the Electro Mechanics Module was added to the previous modules. The Maxwell equation for electrostatic charges with differential potential applied between electrodes and insulation on the walls as boundary condition has been solved. Fig. 3 shows the thermal distribution and the displacement corresponding to $V_{T h}=3.5 \mathrm{~V}$ and $V_{D C}=55 \mathrm{~V}$.

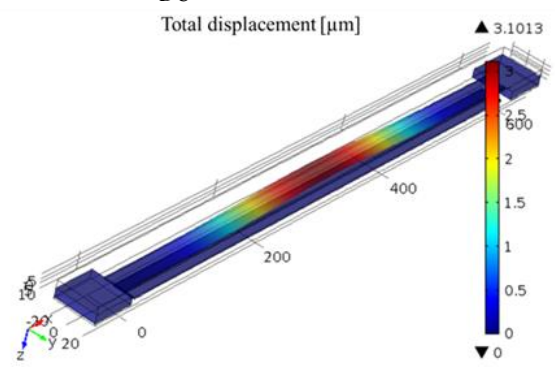

(a)

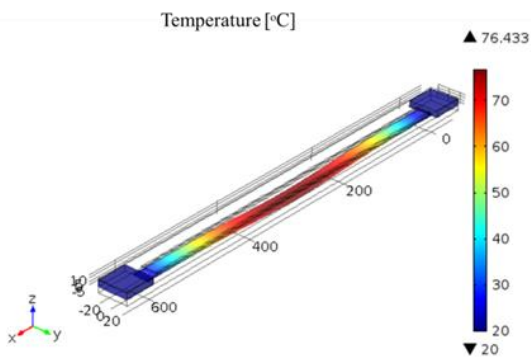

(b)

Fig. 3. (a) Total displacement. (b)Temperature distribution at $V_{T h}=3.5 \mathrm{~V}$ and $V_{D C}=55 \mathrm{~V}$. 


\section{EXPERIMENTAL SETUP}

The experimental investigation was conducted on an in-plane $600 \mu \mathrm{m}$ long clamped-clamped microbeam fabricated from SOI wafers with highly conductive $25 \mu \mathrm{m} \mathrm{Si}$ device layer by MEMSCAP [35]. Micro System Analyzer with in-plane microstructure vibration and motion analysis using stroboscopic video microscopy from Polytec [36], Fig. 4, is used to determine the deflection as well as the resonance frequency of the microbeam.

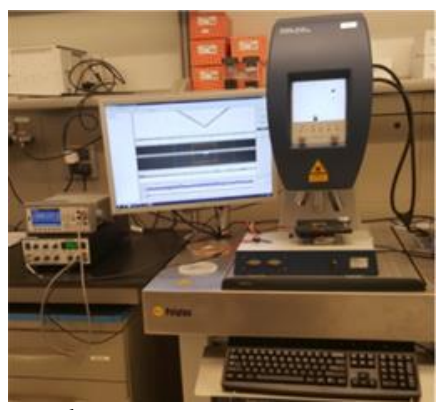

Fig. 4. Experimental setup.

While varying the DC electrothermal voltage, we measured the resonance frequencies of the microbeam using the ring down measurement and the fast Fourier transform (FFT). The ring down measurement is similar to a hammer test, in which we subject the microbeam to a sudden DC electrostatic load that is then removed to allow the microbeam to vibrate freely (ring down) until the motion dies out.

The FFT for a zero electrothermal voltage of the microbeam under consideration is depicted in Fig. 5. The first resonance frequency of the unactuated microbeam is found at $57 \mathrm{kHz}$.

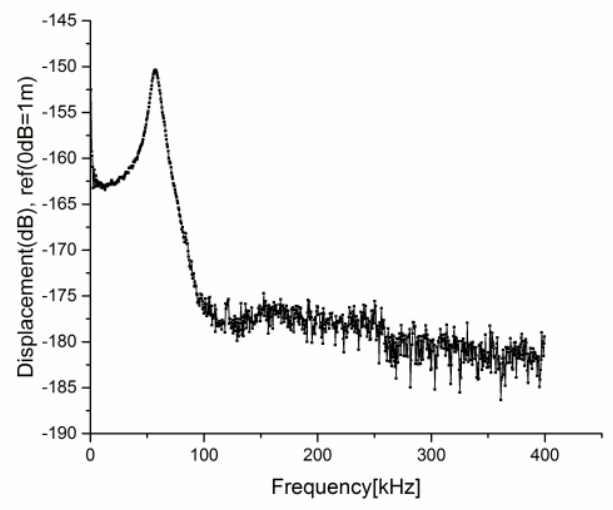

Fig. 5. FFT of the unactuated in-plane clamped-clamped microbeam.
Then, the microbeam under consideration is actuated in addition to the electrothermal actuation electrostatically by adding an external circuit. We applied a constant DC polarization voltage to actuate the microbeam electrostatically and as varying the DC electrothermal voltage we measured the resonance frequency using the ring down measurement.

\section{RESULTS AND DISCUSSION}

The microbeam is first actuated electrothermally without including the electrostatic force. The electrothermal voltage $V_{T h}$ is increased from small values, and therefore the compressive stress inside the microbeam increases. The exact solution of the static deflection due to the electrothermal actuation is given by the first buckling configuration (A3). Experimentally, a topographical characterization, through light interferometry, is used to determine the static deflection of the mid-point of the microbeam as the electrothermal voltage varies. Fig. 6 shows the measured and the simulated mid-point deflection of the microbeam due to electrothermal actuation without the electrostatic force demonstrating the critical buckling limit. After this limit, the microbeam is no longer straight and it is buckled. Fig. 6 demonstrates that the microbeam encounters a pitchfork bifurcation at the critical buckling limit that describes the required voltage to buckle the microbeam. A good agreement is shown among the analytical, finite element, and experimental results.

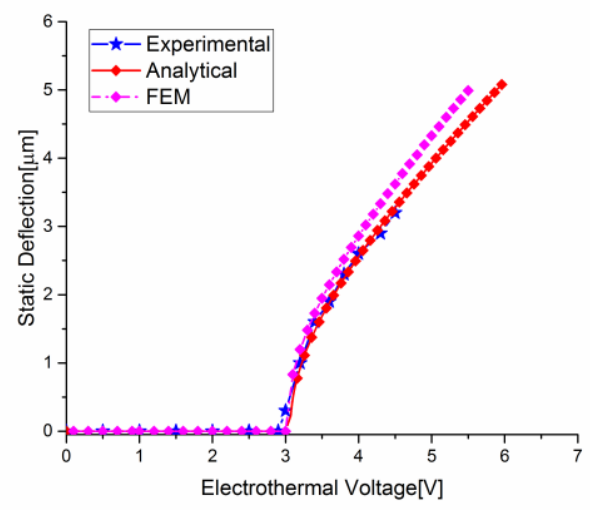

Fig. 6. The mid-point deflection of the microbeam under electrothermal actuation.

The variation of the first resonance frequency of the studied resonator as varying the electrothermal actuation voltage without including the electrostatic force is shown in Fig. 7. The pre-buckling eigenvalue is determining by solving the eigenvalue problem of the straight microbeam under axial load presented in 
(13). For the buckling problem, the eigenvalue problem of buckled beam presented in the appendix is solved. As shown in Fig. 7, the frequency decreases from the initial resonance frequency to almost zero before buckling. After buckling, the fundamental frequency increases from zero to higher values, which can be as high as double the original resonance frequency. In that case, the behavior of the beam is transformed from straight to buckle beam. A good agreement is shown among the analytical, finite element, and experimental results.

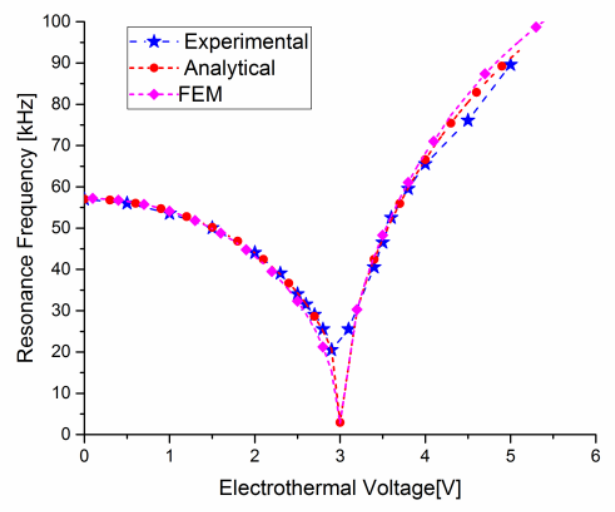

Fig. 7. The first resonance frequency of the microbeam under electrothermal actuation with no DC electrostatic polarization voltage.

Figure 8 demonstrates operating the microbeam as a resonator, while electrothermally actuated and electrostatically excited into vibration, by showing two frequency response curves before and after buckling. The response of both cases is linear around the first resonance frequency with amplitude of vibration in the order of one micrometer. As noted, the response looks stable and above noise level.

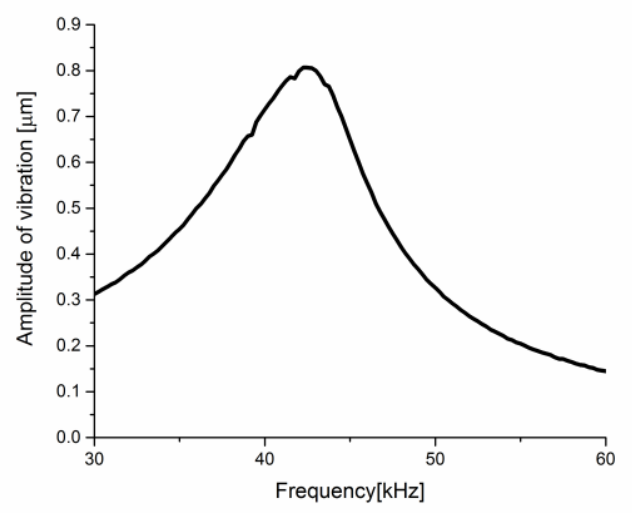

(a)

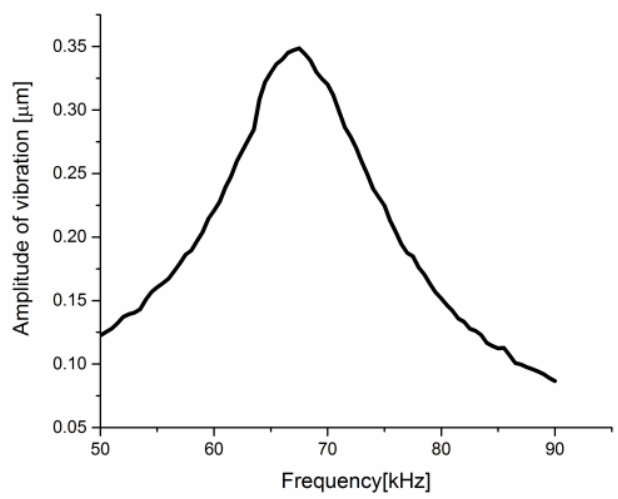

(b)

Fig. 8. Frequency response curves of the studied microbeam, (a) before buckling for $V_{T h}=1 \mathrm{~V}, V_{D C}=40 \mathrm{~V}$ and $V_{A C}=6 \mathrm{~V}$, (b) after buckling for $V_{T h}=4 \mathrm{~V}, V_{D C}=20 \mathrm{~V}$, and $V_{A C}=20 \mathrm{~V}$.

Next, we combined the electrothermal actuation with the electrostatic force. We applied a constant DC polarization voltage, far from the pull-in voltage, between the microbeam and the stationary electrode, and then we varied the electrothermal voltage. The analytical results of the static deflection of the midpoint of the microbeam as varying the electrothermal voltage for a fixed DC polarization voltage is shown in Fig. 9. The static deflection is obtained by solving (11), before the critical load, and (33), after exceeding the critical load. Fig. 9 shows that the microbeam encounters a perturbed pitchfork bifurcation, where the discontinuity in the deflection curve observed in Fig. 6 disappears.

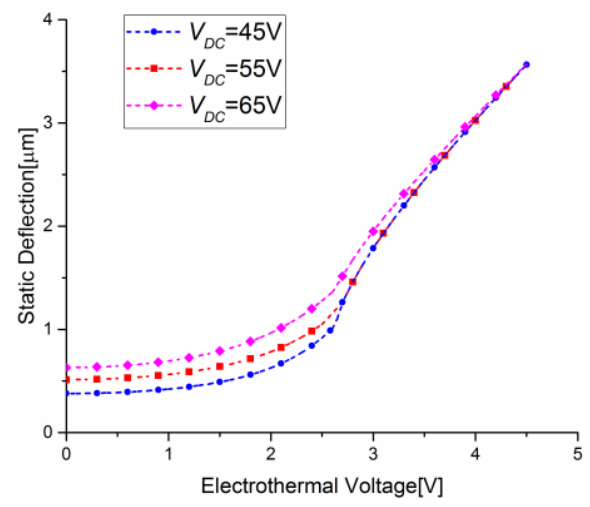

Fig. 9. The static deflection versus electrothermal actuation voltage for a constant DC electrostatic polarization voltage.

Then, solving the system of algebraic equations (18), for the pre-buckling behavior and the system of algebraic equations (24) for the post-buckling behavior, we compute the first natural frequency of the microbeam under constant $V_{D C}$ and as varying $V_{T h}$. Figures10.a, 10.b and 10.c display the first 
resonance frequency of the microbeam versus the electrothermal actuation for a DC electrostatic polarization voltage equal to $45 \mathrm{~V}, 55 \mathrm{~V}$ and $65 \mathrm{~V}$, respectively. A good agreement is shown among the analytical, finite element, and experimental results.

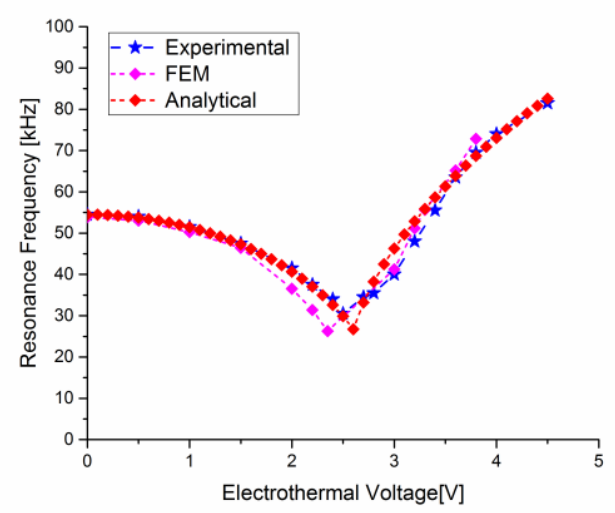

(a)

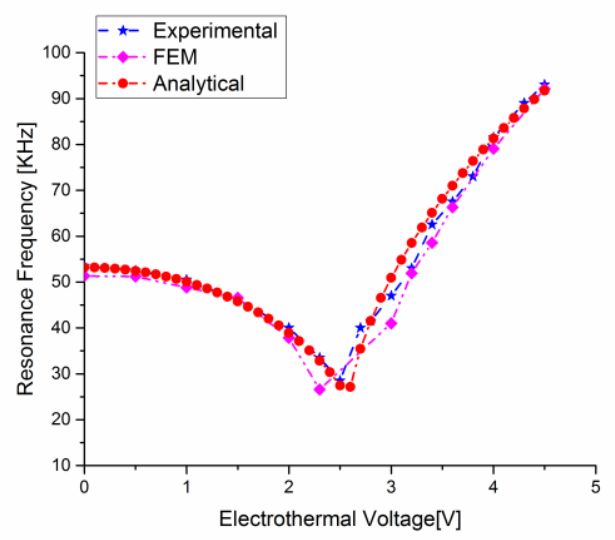

(b)

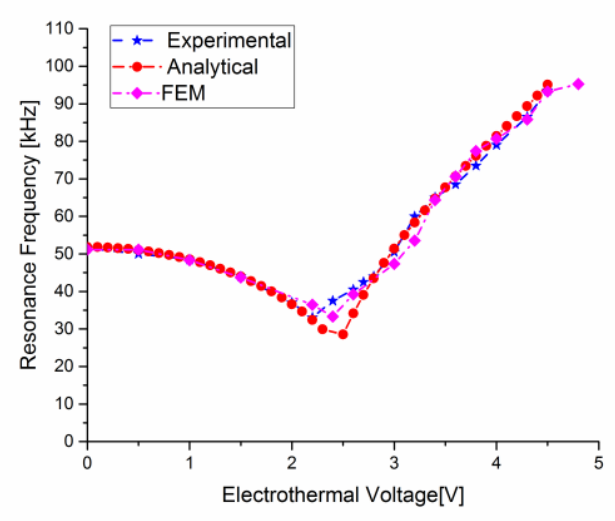

(c)

Fig. 10. The first resonance frequency of the microbeam versus electrothermal actuation voltage for a constant DC electrostatic polarization voltage. (a) $V_{D C}=45 \mathrm{~V}$. (b) $V_{D C}=55 \mathrm{~V}$. (c) $V_{D C}=65 \mathrm{~V}$.
One can note that adding a DC electrostatic polarization changes the qualitative nature of the tunability both before and after buckling, which adds another independent way of tuning. Fig. 11 further clarifies this aspect. The figure shows the resonance frequency, computed analytically, while varying the electrothermal voltage for different values of DC polarization voltages. Adding a DC electrostatic polarization reduces the dip in the resonance frequency before buckling, and can eliminate it if desired with increasing the DC polarization voltage, and further enhances the increase in the resonance frequency after buckling. Additionally, Fig. 11 displays that the initial natural frequency, at zero electrothermal voltage, increases as the DC voltage exceeds $65 \mathrm{~V}$. This fact is due to the dominating effect of mid-plane stretching over electrostatic force for large gaps, as shown in previous works $[27,37]$.

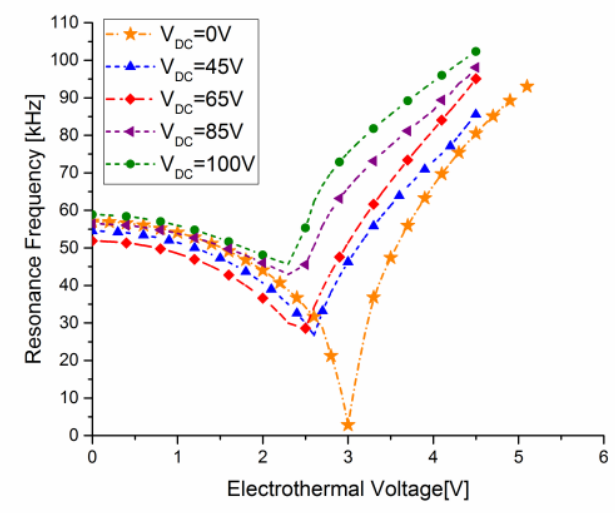

Fig. 11. The first resonance frequency of the microbeam versus electrothermal actuation voltage for different constant DC electrostatic polarization voltages.

One can note from Fig. 11 that the electrothermal tuning has resulted in a considerable increase or decrease in the natural frequency compared to its initial value. The results show a decrease of up to $64.9 \%$ before buckling and then an increase up to $66.6 \%$ of the natural frequency after buckling (compared to the initial value at $V_{T h}=0$ ). This is remarkable compared to the reported works on electrothermal actuation, which showed only decrease in frequency, in [21] $(6.5 \%)$ and in [22] $(42.6 \%)$.

\section{CONCLUSION}

In this paper, we investigated the tunability of the in-plane clamped-clamped microbeam actuated electrothermally and electrostatically using the analytical and numerical methods (FEM) as well as the experimental results. We compared these results to both the experimental and the simulated data. A 
good agreement is shown among analytical, experimental, and numerical results. At first we studied the static deflection and the variation of the first natural frequency of the microbeam under only electrothermal actuation. We showed that as increasing the DC electrothermal voltage the microbeam buckles after a certain critical electrothermal voltage and it is no longer straight beam. Before buckling, the fundamental frequency decreases until the resonance frequency drops to very low values (almost zero). After buckling, the resonance frequency increases to high values, which can reach the double of the original resonance frequency. This has been achieved using very small electrothermal voltage (ranges from 0-5 V).

Then, we added a DC electrostatic polarization voltage in addition to the electrothermal actuation. The microbeam encounters a perturbed pitchfork bifurcation due to DC polarization voltage. We showed that the dip in the resonance frequency before bucking is reduced and the resonance frequency after buckling is increased as increasing the DC polarization voltage. In conclusion, we demonstrate that a single resonator electrothermally and electrostatically actuated can be operated at a wide range of resonance frequency, as low as almost zero frequency to as high as twice of its unactuated resonance frequency by only controlling the electrothermal and the electrostatic voltages.

\section{APPENDIX: POST-BUCKLING STUDY}

Here, we consider the buckling problem of the beam under the compressive load without electrostatic forces. We follow in the derivation here the work of Nayfeh et al [32, 33]. The equation governing the static configuration in the buckled position $\psi(x)$ can be written as

$$
\psi^{i v}+P \psi^{\prime \prime}-\alpha_{1} \psi^{\prime \prime} \int_{0}^{1} \psi^{\prime 2} d x=0
$$

where $P=S_{T h}-N_{0}$ represents the total axial load. (A1) is subjected to the following boundary conditions

$$
\begin{aligned}
& \psi(0)=\psi(1)=0 \\
& \left.\frac{d \psi}{d x}\right|_{x=0}=\left.\frac{d \psi}{d x}\right|_{x=1}=0
\end{aligned}
$$

The analytical solution of the first buckling configuration is given by

$$
\psi(x)=\frac{1}{2} b_{1}[1-\cos (2 \pi x)]
$$

where $b_{1}$ is the rise at midpoint of the microbeam of the first buckling mode of the clamped-clamped microbeam and is given by the following expression:

$$
b_{1}=\sqrt{\frac{2\left[P-4 \pi^{2}\right]}{\alpha_{1} \pi^{2}}}
$$

To compute the natural frequencies and the mode shape of the buckled beam, we solve the eigenvalue problem governing the mode shape of the buckled unactuated beam governed by $[29,30]$ :

$$
\begin{gathered}
\varphi^{i v}+4 \pi^{2} \varphi^{\prime \prime}-4 \alpha_{1} b_{1}^{2} \pi^{3} \cos (2 \pi x) \int_{0}^{1} \varphi^{\prime} \sin (2 \pi x) d x \\
=\omega^{2} \varphi^{\prime \prime}
\end{gathered}
$$

where $\omega$ is associated nondimensional frequency. (A5) is subjected to the below boundary condition:

$$
\begin{aligned}
& \varphi(0)=\varphi(1)=0 \\
& \left.\frac{d \varphi}{d x}\right|_{x=0}=\left.\frac{d \varphi}{d x}\right|_{x=1}=0
\end{aligned}
$$

To solve (A5), we set $\varphi$ equal to the superposition of a homogeneous solution $\varphi_{h}$ and a particular solution $\varphi_{p}$, hence we let

$$
\varphi(x)=\varphi_{h}(x)+\varphi_{p}(x)
$$

The general solution of (A5) that represents the mode shapes of the buckled beam is given by

$$
\begin{aligned}
& \varphi(x)=c_{1} \cos \left(s_{1} x\right)+c_{2} \sin \left(s_{1} x\right)+c_{3} \cosh \left(s_{2} x\right) \\
& +c_{4} \sinh \left(s_{2} x\right)+c_{5} \cos (2 \pi x)
\end{aligned}
$$

where $s_{1}$ and $s_{2}$ are defined as below

$$
s_{1,2}=\sqrt{ \pm 2 \pi^{2}+\sqrt{4 \pi^{2}+\omega^{2}}}
$$

The $c_{\mathrm{i}}$ are constants determined by substituting (A8) into the boundary conditions (A6) and (A7) into (A5) using the fact that $\varphi_{p}(x)=c_{5} \cos (2 \pi x)$. This yields five algebraic equations for the coefficients $c_{\mathrm{i}}$ and the natural frequency $\omega$. For a fixed level of $b_{1}$, the symmetric natural frequencies as well as their corresponding mode shapes are found by setting the determinant of the coefficient matrix equal to zero.

\section{REFERENCES}

[1] R. Harne and K. Wang, "A review of the recent research on vibration energy harvesting via bistable systems," Smart Materials and Structures, vol. 22, p. 023001, 2013.

[2] A. Nikitin, N. G. Stocks, and A. Bulsara, "Bistable sensors based on broken symmetry phenomena: The residence time difference vs. the second harmonic method," The European Physical Journal Special Topics, vol. 222, pp. 2583-2593, 2013.

[3] P. Chouinard and J.-S. Plante, "Bistable antagonistic dielectric elastomer actuators for binary robotics and 
mechatronics," Mechatronics, IEEE/ASME Transactions on, vol. 17, pp. 857-865, 2012.

[4] B. Charlot, W. Sun, K. Yamashita, H. Fujita, and H. Toshiyoshi, "Bistable nanowire for micromechanical memory," Journal of Micromechanics and Microengineering, vol. 18, p. 045005, 2008.

[5] H. Maekoba, P. Helin, G. Reyne, T. Bourouina, and H. Fujita, "Self-aligned vertical mirror and V-grooves applied to an optical-switch: modeling and optimization of bi-stable operation by electromagnetic actuation," Sensors and Actuators A: Physical, vol. 87, pp. 172178, 2001.

[6] M. Notomi, A. Shinya, S. Mitsugi, G. Kira, E. Kuramochi, and T. Tanabe, "Optical bistable switching action of Si high-Q photonic-crystal nanocavities," Optics Express, vol. 13, pp. 2678-2687, 2005.

[7] X. Liu, H. A. Hajjar, F. Lamarque, E. Dore, O. Carton, A. Zeinert, et al., "An optical wireless bistable microactuator," in Mechatronics and Automation (ICMA), 2015 IEEE International Conference on, 2015, pp. 1624-1629.

[8] D. Zaccaria, D. Bigoni, G. Noselli, and D. Misseroni, "Structures buckling under tensile dead load," in Proceedings of the Royal Society of London A: Mathematical, Physical and Engineering Sciences, 2011, p. rspa20100505.

[9] J. M. Maloney, D. S. Schreiber and D. L. DeVoe, "Large-force electrothermal linear m micromotors", Journal of Micromechanics and Microengineering, 14:226-234, 2004.

[10] M. Chiao and L. Lin, "Self-buckling of micromachined beams under resistive heating," Microelectromechanical Systems, Journal of, vol. 9, pp. 146-151, 2000.

[11] Y. Wang, Z. Li, D. T. McCormick, and N. C. Tien, "A low-voltage lateral MEMS switch with high RF performance," Microelectromechanical Systems, Journal of, vol. 13, pp. 902-911, 2004.

[12] X. Chen, L. Ma, Y. Zheng, and D.-W. Lee, "Theoretical analysis of postbuckling behavior with experimental validation using electrothermal microbeams," Applied Physics Letters, vol. 98, p. 073107, 2011.

[13] E. Mastropaolo and R. Cheung, "Electrothermal actuation studies on silicon carbide resonators," Journal of Vacuum Science \& Technology B, vol. 26, pp. 26192623, 2008.

[14] T. Sibgatullin, D. Schreiber, and S. Krylov, "Excitation of parametric resonance in micro beams by Joule's heating," Proc. ofCOMDYN 2013:4 $4^{\text {th }}$ ECCOMAS Thematic Conferene on Computational Methods in Structural Dynamics And Earthquaque Engineering , Kos Island, Greece, Jun. 12-14, 2013

[15] D. Girbau, A. Lázaro, A. Pérez, E. Martínez, L. Pradell, and R. Villarino, "Tunable dual-band resonators for communication systems," International Journal of Microwave and Wireless Technologies, vol. 2, p. 245, 2010

[16] S. Sirci, J. Martinez, M. Taroncher, and V. Boria, "Varactor-loaded continuously tunable SIW resonator for reconfigurable filter design," in Microwave Conference (EuMC), 2011 41st European, 2011, pp. 436-439.

[17] S. Sonmezoglu, S. E. Alper, and T. Akin, "An automatically mode-matched MEMS gyroscope with wide and tunable bandwidth," Microelectromechanical Systems, Journal of, vol. 23, pp. 284-297, 2014.

[18] C. Peters, D. Maurath, W. Schock, F. Mezger, and Y. Manoli, "A closed-loop wide-range tunable mechanical resonator for energy harvesting systems," Journal of
Micromechanics and Microengineering, vol. 19, p. 094004, 2009.

[19] L. Zhuang, W. Beeker, A. Leinse, R. Heideman, and C. Roeloffzen, "Continuously tunable photonic fractional Hilbert transformer using ring resonators for on-chip microwave photonic signal processing," in Microwave Photonics (MWP), 2012 International Topical Meeting on, 2012, pp. 22-25.

[20] J. Moser, J. Güttinger, A. Eichler, M. J. Esplandiu, D. Liu, M. Dykman, et al., "Ultrasensitive force detection with a nanotube mechanical resonator," Nature nanotechnology, vol. 8, pp. 493-496, 2013.

[21] T. Remtema and L. Lin, "Active frequency tuning for micro resonators by localized thermal stressing effects," Sensors and Actuators A: Physical, vol. 91, pp. 326332, 2001.

[22] H. Goktas and M. E. Zaghloul, "Tuning In-Plane FixedFixed Beam Resonators With Embedded Heater in CMOS Technology," Electron Device Letters, IEEE, vol. 36, pp. 189-191, 2015.

[23] B. Sviličić, E. Mastropaolo, B. Flynn, and R. Cheung, "Electrothermally actuated and piezoelectrically sensed silicon carbide tunable MEMS resonator," Electron Device Letters, IEEE, vol. 33, pp. 278-280, 2012.

[24] S. C. Jun, X. Huang, M. Manolidis, C. Zorman, M. Mehregany, and J. Hone, "Electrothermal tuning of AlSiC nanomechanical resonators," Nanotechnology, vol. 17, p. 1506, 2006.

[25] H. C. Nathanson, W. E. Newell, R. Wickstrom, and J. R. Davis Jr, "The resonant gate transistor," Electron Devices, IEEE Transactions on, vol. 14, pp. 117-133, 1967.

[26] A. H. Nayfeh, M. I. Younis, and E. M. Abdel-Rahman, "Reduced-order models for MEMS applications," Nonlinear dynamics, vol. 41, pp. 211-236, 2005.

[27] E. M. Abdel-Rahman, M. I. Younis, and A. H. Nayfeh, "Characterization of the mechanical behavior of an electrically actuated microbeam," Journal of Micromechanics and Microengineering, vol. 12, p. 759, 2002.

[28] V. Sazonova, Y. Yaish, H. Üstünel, D. Roundy, T. A. Arias, and P. L. McEuen, "A tunable carbon nanotube electromechanical oscillator," Nature, vol. 431, pp. 284-287, 2004.

[29] H. M. Ouakad and M. I. Younis, "Modeling the Structural-Thermal-Electrical Coupling in an Electrostatically Actuated MEMS Switch and Its Impact on the Switch Stability," Mathematical Problems in Engineering, Vol. 2013, pp. 1-8.

[30] M. I. Younis," MEMS linear and nonlinear statics and dynamics," vol. 20: Springer Science \& Business Media, 2011.

[31] M. I. Younis, EM. Abdel-Rahman, A. H. Nayfeh, "A reduced-order model for electrically actuated microbeam-based MEMS," Microelectromechanical Systems, Journal of. 2003;12(5):672-80.

[32] A. H. Nayfeh, W. Kreider, and T. Anderson, "Investigation of natural frequencies and mode shapes of buckled beams," AIAA journal, vol. 33, pp. 11211126, 1995.

[33] A. H. Nayfeh and P. F. Pai, Linear and nonlinear structural mechanics: John Wiley \& Sons, 2008.

[Online]. https://www.comsol.com/
[Online].Polytech: http://www.polytec.com/us/. A. Z. Hajjaj, A. Ramini, and M. I. Younis, "Experimental and analytical study of highly tunable electrostatically actuated resonant beams," Journal of Micromechanics and Microengineering, vol. 25, p. 125015, 2015. 


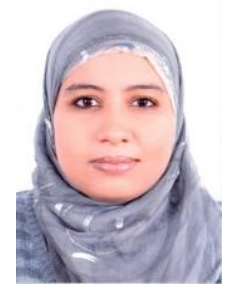

Amal Z. Hajjaj received a B.S. and Master's degrees in Mechanical Engineering from Tunisia Polytechnic School in 2012 and 2013. She is currently enrolled as a PhD candidate in Mechanical Engineering in King Abdullah University of Science and Technology (KAUST), Thuwal, Saudi Arabia. She is interested in characterizing theoretically and experimentally the linear and nonlinear dynamics of MEMS based resonators with their applications in MEMS sensors and actuators.

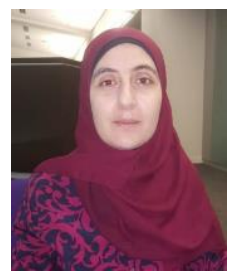

Nouha Alcheikh was born in 1984. She received her MS degree in electronics from the Polytechnic National Institute of Grenoble in 2007 and her PhD degree in RF MEMS from Grenoble University, France in 2011. From 2011 to 2014, she was as a PostDoctoral fellow working on Force Sensors and Energy Harvesting at CEALeti/MINATEC Campus, Grenoble (France) and at IMS, Bordeaux (France). Since 2015, she has been a Post-Doctoral Fellow at King Abdullah University of Science and Technology, Thuwal, Saudi Arabia where she is performing her research on MEMS Sensors and Actuators.

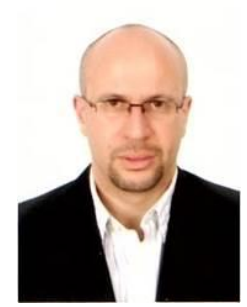

Abdallah Ramini was born in Amman, Jordan, in 1981. He received the Bachelor and Master's degrees from Jordan University of Science and Technology in Mechatronics Engineering, Jordan in 2004 and 2007, respectively; and the Ph.D. degree in Mechanical Engineering from Binghamton State University of New York in 2012, with a focus on the static and dynamic behaviors of MEMS devices and structures under shock. From 2012 to 2013, he was with Binghamton State University of New York as a Post-Doctoral Fellow working on dynamical integrity of MEMS resonators. Since 2013, he has been a PostDoctoral Fellow at King Abdullah University of Science and Technology in Saudi Arabia. Currently, he is performing his research activity with MEMS and NEMS Characterization and Motion Laboratory. His research focuses on MEMS resonant and actuator applications. Dr. Ramini is a member of the American Society of Mechanical Engineers ASME.

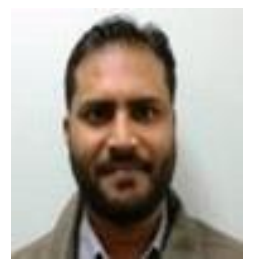

Md. Abdullah Al Hafiz received his B.Sc. (Engg) degree in Electrical and Electronic Engineering from the Islamic University of Technology (IUT), Dhaka, Bangladesh, in 2001. From April 2003 to February 2006, he was a lecturer in the Department of EEE at the Rajshahi University of Engineering and Technology (RUET), Rajshahi, Bangladesh. In February 2011, he received his Ph.D. degree in Electrical Engineering from the
University of New South Wales (UNSW), Sydney, Australia. Currently, he is working as Post-doc Fellow at KAUST in the area of using MEMS resonators for tunability and for logic and memory applications. His research interests are MEMS/MOEMS systems, planar light wave technologies, interconnects, optical crossconnects, and MEMS resonators.

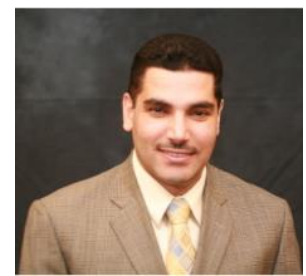

Mohammad I. Younis received the B.S. degree in mechanical engineering from the Jordan University of Science and Technology, Irbid, Jordan, in 1999, and the M.S. and Ph.D. degrees in engineering mechanics from Virginia Polytechnic Institute and State University, Blacksburg, VA, USA, in 2001 and 2004, respectively. He is currently an Associate Professor of Mechanical Engineering with the King Abdullah University of Science and Technology, Thuwal, Saudi Arabia, and the State University of New York (SUNY), Binghamton, NY, USA. He serves as the Director of the MEMS and NEMS Characterization and Motion Laboratory. Dr. Younis is a recipient of the SUNY Chancellor's Award for Excellence in Scholarship and Creative Activities in 2012, the National Science Foundation Faculty Early Career Development Award in 2009, and the Paul E. Torgersen Graduate Research Excellence Award in 2002. He holds several U.S. patents in MEMS sensors and actuators. He serves as an Associate Editor of Nonlinear Dynamics, the Journal of Computational and Nonlinear Dynamics, and the Journal of Vibration and Control. He has authored the book entitled MEMS Linear and Nonlinear Statics and Dynamics (Springer, 2011). He is a member of the American Society of Mechanical Engineers. 